\title{
Surgical Orbital Anatomy
}

\author{
Shirley Hu, MD ${ }^{1,2}$ Patrick Colley, $\mathrm{MD}^{1,2}$ \\ ${ }^{1}$ Department of Otolaryngology, Mount Sinai Medical Center, New \\ York, New York \\ 2 Department of Otolaryngology, New York Eye and Ear Infirmary of \\ Mount Sinai, New York, New York
}

Address for correspondence Shirley Hu, MD, 310 East 14th Street, New York, New York 10003 (e-mail: shu@nyee.edu).

Semin Plast Surg 2019;33:85-91.

\begin{abstract}
Keywords

- orbital anatomy

- surgical

- globe

In this article, the anatomy of the orbit is reviewed, with a specific emphasis on surgical anatomy. A brief discussion of the ocular globe is also included. The orbits are pyramidal structures separating the upper and middle facial skeletons. The walls, apex, and base harbor several foramina and fissures as well as bony irregularities where various ligaments, muscles, and capsules attach. There are a variety of surgical approaches to the orbit, including the traditional transcutaneous and neurosurgical techniques and, more recently, minimally invasive, endoscopic approaches.
\end{abstract}

The orbit is a pyramidal structure that encompasses the organ of vision and separates the upper and middle facial skeletons, with its apex located posteriorly and base situated anteriorly. The bone comprising the apex and base is much thicker than that of the walls, allowing the apex to protect the brain and optic nerve from direct force and the orbital rim to resist fracture. Pressure to the globe is thus dispersed to the curvilinear orbital walls, which serve to maintain the projection of the ocular globe and cushion it from blunt force.

The height of the orbit is usually $35 \mathrm{~mm}$, and the width from rim to rim is approximately $40 \mathrm{~mm}$. The distance from the medial orbital rim to the apex averages $45 \mathrm{~mm}$ in length, while the distance from the lateral orbital rim to the apex is approximately $1 \mathrm{~cm}$ shorter. ${ }^{1,2}$

\section{Bony Topography}

Seven bones comprise the orbit (-Fig. 1). The lateral wall is formed by the greater wing of the sphenoid, the frontal bone, and the zygomatic bone. The floor is composed of the sphenoid, the orbital process of the palatine bone, and the orbital process of the maxillary bone. The medial wall is formed from the lesser wing of the sphenoid, the ethmoid bone, the lacrimal bone, and the frontal process of the maxilla. The roof of the orbit is derived from the sphenoid and the frontal bones. ${ }^{1-6}$

\section{Floor of the Orbit}

From the inferior orbital rim, the floor dips inferiorly, maintains the same cephalocaudad position for approximately $15 \mathrm{~mm}$ beyond the inferior orbital fissure, and then gently curves up toward the superior orbital fissure. When repairing orbital floor fractures, recreating this subtle curvature will restore normal anatomy and help prevent malpositioning of the globe. ${ }^{7}$

\section{Medial Orbital Wall}

The medial orbital wall is in the sagittal plane and has the greatest degree of cephalocaudad curvature. It separates the ethmoid sinuses and nasal cavity from the orbit. The superior aspect of the medial rim forms the forehead, curving anteriorly toward the midline. The bone of the medial orbital wall is thin throughout but strengthened by the perpendicular septa of the ethmoid sinus.

\section{Roof and Lateral Orbital Wall}

The roof separates the anterior cranial fossa from the orbit. The medial third of the superior orbital rim harbors a notch or foramen through which the supraorbital nerve, artery, and vein traverse, providing blood supply and sensation to the forehead. Additionally, the supratrochlear nerve exits just medial to the supraorbital nerve in between the pulley of the superior oblique and the supraorbital notch or foramen. The supraorbital nerve arises an average of $29 \mathrm{~mm}$ lateral to the midline, while the supratrochlear nerve is located $16 \mathrm{~mm}$ lateral to midline. ${ }^{8,9}$ The lateral roof of the orbit then curves superiorly to house the lacrimal gland.

The lateral orbital rim has the least projection, which facilitates lateral vision. The zygomatic portion of the lateral orbital wall is relatively fragile, but thickens considerably as
Issue Theme Orbital Reconstruction; Guest Editor: Sameep Kadakia, MD
Copyright (c) 2019 by Thieme Medical Publishers, Inc., 333 Seventh Avenue, New York, NY 10001, USA. Tel: +1(212) 584-4662.
DOI https://doi.org/ 10.1055/s-0039-1685476. ISSN 1535-2188. 


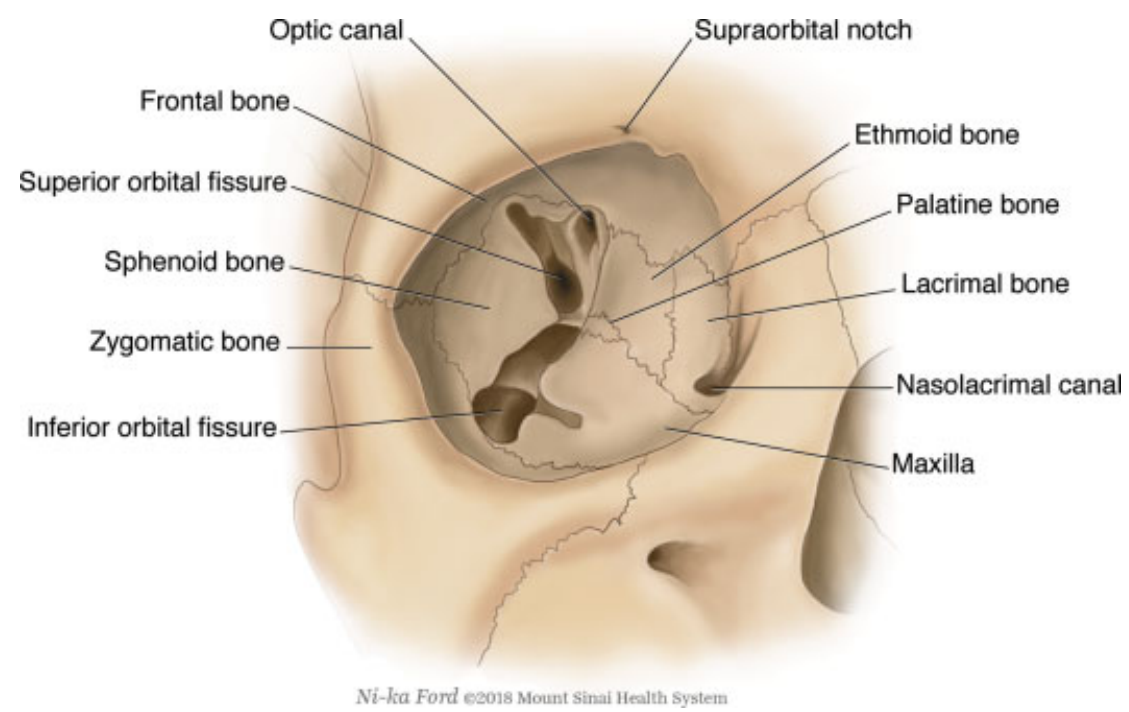

Fig. 1 The seven bones that comprise the bony orbit.

it nears the sphenoid bone, where it borders the superior orbital fissure.

\section{Foramina, Fissures, Crests, and Their Neurovascular Components}

There are three main conduits into and out of the bony orbit (-Fig. 2): (1) the optic canal, which transmits the optic nerve; (2) the superior orbital fissure, which transmits the lacrimal, frontal, trochlear, oculomotor, nasociliary, and abducens nerves as well as the superior ophthalmic vein; and (3) the inferior orbital fissure, through which the maxillary nerve, the inferior ophthalmic vein, and sympathetic nerves traverse.

\section{Optic Canal}

The optic canal is approximately $5 \mathrm{~mm}$ in diameter and runs in a superomedial direction to enter the cranial fossa. The bony canal itself is less than $1 \mathrm{~cm}$ in length and is in the medial portion of the orbital apex, lying entirely within the sphenoid bone. The extent of the sphenoid sinus pneumatization may lead to thinning of the canal or the optic nerve coursing through the lateral portion of the sinus. Lesions or fractures affecting the optic canal can cause orbital apex syndrome, which is characterized by ophthalmoplegia, hypoesthesia, and blindness.

\section{Superior Orbital Fissure}

The superior orbital fissure is superolateral to the optic canal and extends inferiorly to a point just medial to this canal. It is formed by the cleft between the greater and lesser wings of the sphenoid. Fractures, edema, or infection extending to the superior orbital fissure can result in superior orbital fissure syndrome, which is marked by ophthalmoplegia, ptosis, and pupillary dilatation.

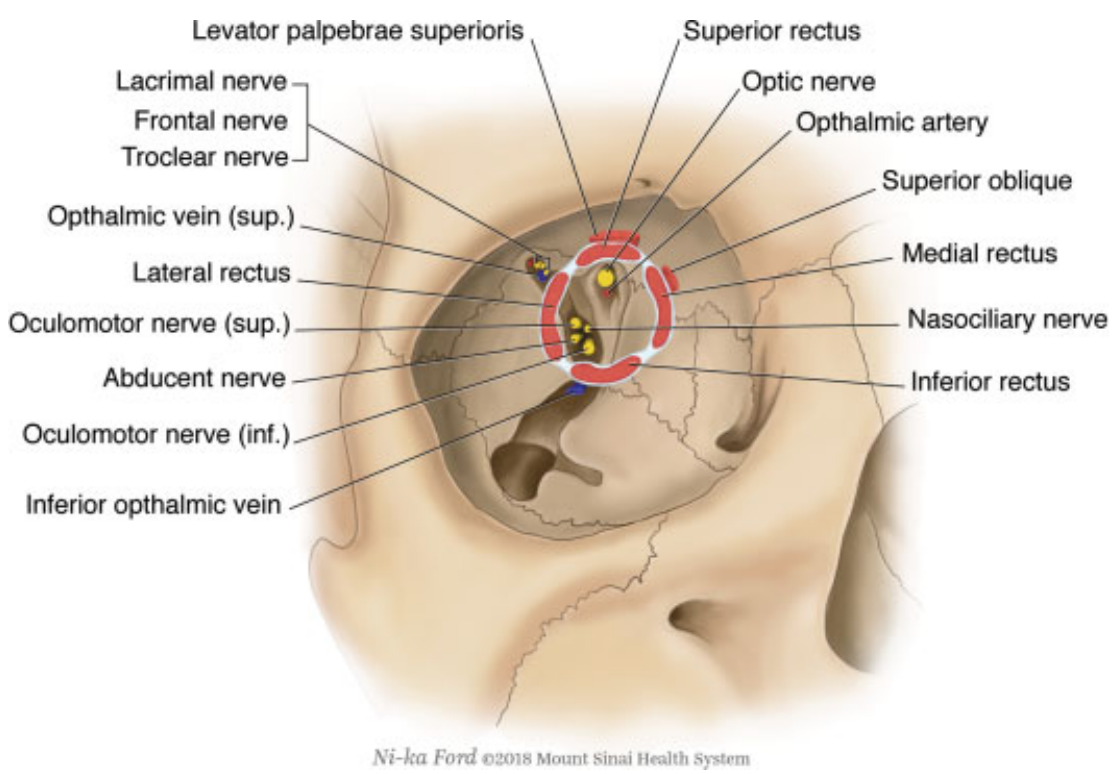

Fig. 2 Foramina, fissures, and their neurovascular components. 


\section{Inferior Orbital Fissure}

The inferior orbital fissure is the inferolateral continuation of superior orbital fissure. It is formed by the zygoma and greater wing of the sphenoid laterally and the zygoma and maxilla medially. The fissure connects the floor of the orbit with the pterygopalatine and infratemporal fossae. In the anterior portion of the fissure, a small canal runs forward through the floor of the orbit and exits the maxilla approximately $5 \mathrm{~mm}$ inferior to the rim. This canal carries the infraorbital nerve, which also gives off the anterior, superior alveolar, and middle superior alveolar nerves. The infraorbital nerve is accompanied by its artery, a terminal branch of the internal maxillary artery, and vein, which drains into the pterygoid plexus.

\section{Anterior and Posterior Ethmoidal Foramina}

The anterior and posterior ethmoidal foramina exit between the frontal and ethmoid bones at the frontoethmoidal suture. The anterior foramen is approximately $24 \mathrm{~mm}$ behind the anterior lacrimal crest, and the posterior foramen is $12 \mathrm{~mm}$ behind this. The anterior ethmoidal artery enters the anterior cranial fossa through the anterior ethmoidal canal and then the nasal cavity through the cribriform plate. It is accompanied by the anterior ethmoidal nerve, a sensory branch of the nasociliary nerve.

\section{Cranio-Orbital Foramen}

The cranio-orbital foramen is present in 55 to $70 \%$ of all orbits and is located anterior to the superior orbital fissure in the medial orbital wall. It carries within it a branch of the middle meningeal artery that forms an anastomosis with the lacrimal artery. During optic nerve decompression or orbital dissection, the surgeon must remain cognizant of this foramen and its potential for hemorrhage. ${ }^{10}$

\section{Nasolacrimal Canal}

The nasolacrimal canal drains tears from the eye to the nasal cavity and is located in the medial wall of the orbit. The anterior portion of the canal is known as the anterior lacrimal crest and is formed by the maxilla. Posteriorly, the canal is formed by the lacrimal bone and is called the posterior lacrimal crest. The deeper fibers of the medial canthus and orbicularis oculi attach to the posterior lacrimal crest. ${ }^{11}$

\section{Eyelids}

The eyelids are soft tissue structures that protect the anterior surface of the globe from injury and aid in tear flow. The anatomical layers of the lid are best understood using a sagittal cross-section through the lid (-Fig. 3). From superficial to deep, the layers are (1) skin and subcutaneous tissue, (2) orbicularis oculi muscle, (3) orbital septum, (4) orbital fat, (5) levator and Müller's muscles (eyelid retractors), (6) tarsus, and (7) conjunctiva. The anatomy of the lower eyelid is analogous to that of the upper eyelid, except the eyelid retractors which consist of the capsulopalpebral fascia and inferior tarsal muscle, of which the former fuses with the orbital septum $5 \mathrm{~mm}$ below the inferior tarsal border. ${ }^{12}$

In reconstruction, the eyelid can be divided surgically into the anterior and posterior lamellae, with the anterior lamella encompassing the skin and orbicularis and the posterior lamella consisting of the tarsus and conjunctiva. ${ }^{13}$

\section{Medial Canthus}

The medial canthal tendon is formed by the fusion of three tendinous arms extending from the anterior and posterior lacrimal crests. The third or superior arm extends from the orbital process of the frontal bone. The arms merge cephalic to the lacrimal sac and then split again into two different arms that attach to the upper and lower tarsal plates via the crura. ${ }^{10}$

\section{Orbital Septum}

The orbital septum is a dense layer of connective tissue lining the orbit that terminates at the periosteum of the orbital rim, forming the arcus marginalis. It marks the anterior boundary

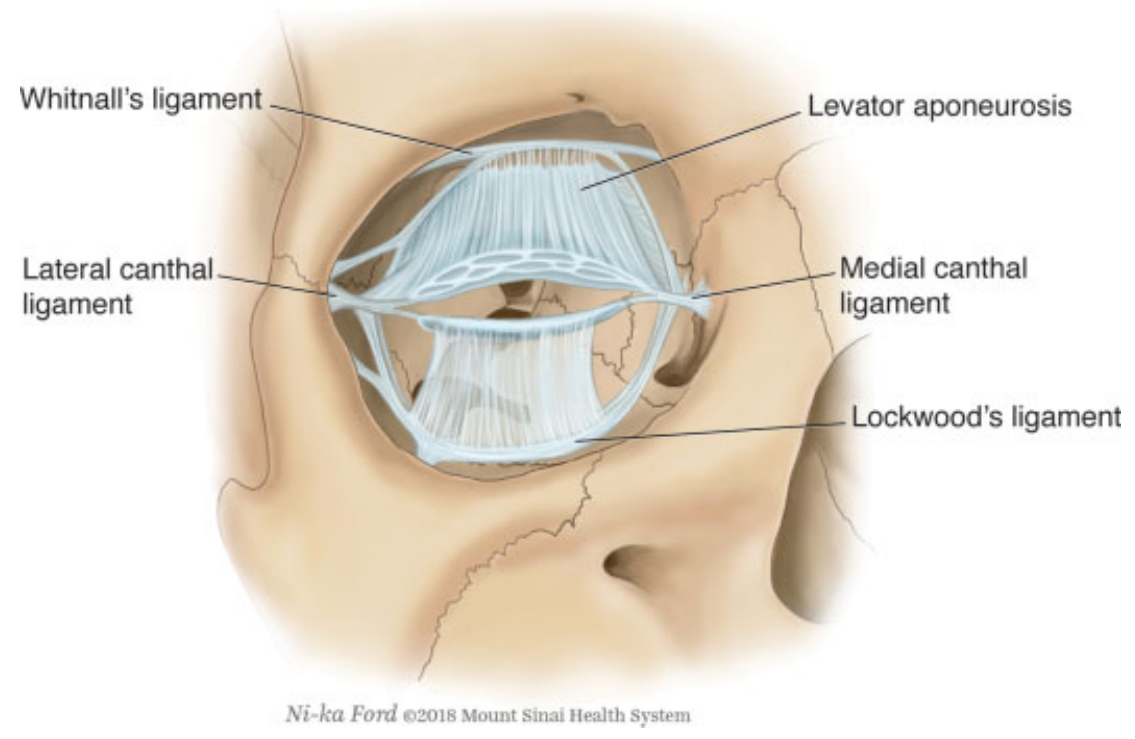

Fig. 3 A sagittal cross-section showing the layers of the upper and lower eyelids. 
of the orbit and is a consistent feature of both the upper and the lower eyelids. In the upper lid, it blends with the tendon of the levator palpebrae superioris and in the lower lid with the capsulopalpebral fascia $5 \mathrm{~mm}$ inferior to the tarsal plate.

\section{Tarsal Plates}

The tarsal plates are curvilinear structures composed of dense connective tissue located directly above the lid margin. The upper tarsal plate is larger, measuring 10 to $12 \mathrm{~mm}$ vertically, while the lower plate measures 3 to $5 \mathrm{~mm}$. They contribute rigidity to the lids and also contain the attachments of multiple muscles and membranes. Specifically, the levator palpebrae superioris attaches to the anterior surface of the superior tarsus; the lateral palpebral raphe extends from the zygomatic bone to the lateral angles of both tarsi; and the medial palpebral ligament extends from the maxilla to the medial angles. Within the tarsal plates are large sebaceous glands, also known as Meibomian glands, which are aligned vertically and secrete a lipid-rich product that prevents lacrimal secretions from evaporating too quickly.

\section{Muscles}

There are several muscles present in the upper and lower eyelids. The orbicularis oculi muscle is present in both and lies just deep to the skin. This muscle can be arbitrarily separated into the orbital and palpebral portions, with the latter being subdivided into the preseptal and pretarsal components. The muscle fibers of the orbital orbicularis originate from the superomedial orbital margin and extend superiorly to interdigitate with the frontalis and corrugator supercilii muscles, laterally to cover the temporalis fascia, and inferiorly to overlie the origins of the lip elevators. The preseptal orbicularis oris overlies the orbital septum, originates medially, and is associated with the medial palpebral ligament. The muscle fibers fuse laterally to form the lateral palpebral raphe, which is then attached to the overlying skin. Underneath this is submuscular areolar tissue, which serves as a potential plane of surgical dissection. The pretarsal portion is located anterior to the tarsus, with a superficial and deep head of origin also associated with the medial palpebral ligament. The fibers course horizontally and laterally deep to the lateral palpebral raphe to insert into the lateral orbital tubercle. The pretarsal portion plays a role in the lacrimal pump.

Underneath the orbital septum in the upper eyelid, the aponeurosis of the levator muscle attaches to the tarsus, and in the lower lid, the fascia of the inferior rectus attaches to the inferior tarsus and then into the orbicularis oculi muscle and subcutaneous tissues of the lid.

Another smooth muscle, the inferior tarsal muscle or Mueller's muscle, arises from the posterior fascia and inserts into the inferior tarsus. Its analogous structure in the upper eyelid is Whitnall's muscle, which is contained in the deep surface of the levator aponeurosis. Both of these smooth muscles are innervated by lacrimal nerve, which carry the sympathetic fibers of the superior cervical ganglion.

\section{Conjunctiva}

The conjunctiva is the deepest layer of the eyelid, forming its inner surface. This reflects on itself from the eyelid at the fornix to cover the outer surface of the eyeball. Sensory innervation is supplied by the ophthalmic branch of the first division of the trigeminal nerve.

\section{Lateral Canthus}

The lateral canthus suspends the tarsus of both lids laterally to the zygomatic bone at Whitnall's tubercle $1.5 \mathrm{~mm}$ inside of the lateral orbital rim. This posterior attachment vector is an important consideration during lid tightening procedures involving the lateral canthal ligament. Also attached to this tubercle are the aponeurosis of the levator and the ligament of the lateral rectus.

\section{Periorbita}

Wherever the cranium and the orbit come into contact (optic canal, superior orbital fissure, anterior and posterior ethmoidal foramina, and the cranio-orbital foramen), the dura becomes continuous with the underlying bone. This layer is known as the periorbita, which is attached loosely to the bone compared to the periosteum elsewhere in the face and cranium. The anterior extension of the periorbita is the orbital septum.

On the orbital surface of the optic canal and the medial aspect of the superior orbital fissure, the periorbita thickens to form the tendinous attachments of the four rectus muscles, the levator superioris muscle, and the superior oblique muscle. This tendinous ring is called the annulus of Zinn.

\section{Ligaments}

The various extraocular ligaments are illustrated in - Fig. 4 .

\section{Tenon's Capsule}

The bulbous sheath or Tenon's capsule is a fibrous layer that extends from the optic nerve to the limbus and envelops the globe, separating it from the intramuscular orbital fat. It fuses with the sheath of the optic nerve, the sclera around the entrance of the optic nerve, and the fascia of the extraocular muscles posteriorly and inferiorly. This capsule forms a socket in which the globe can move. The fascia is traversed by the ciliary vessels and nerves. Tenon's capsule is separated from the outer surface of the sclera by the periscleral lymphatic space, which is continuous with the subdural and subarachnoid cavities. ${ }^{14}$

\section{Lockwood's Ligament}

The lower part of the bulbous sheath is known as the suspensory ligament of Lockwood. This fascial sling blends with the lateral canthus and the lateral check ligament and travels laterally to medially. Medially, the suspensory attachment blends with the medial canthus and the medial check ligament, attaching to the medial orbital wall at the lacrimal crest. The ligament forms a hammock between the lateral and medial orbital margins and encloses the inferior oblique and inferior rectus muscles, providing support and preventing downward displacement of the globe. ${ }^{14}$ 


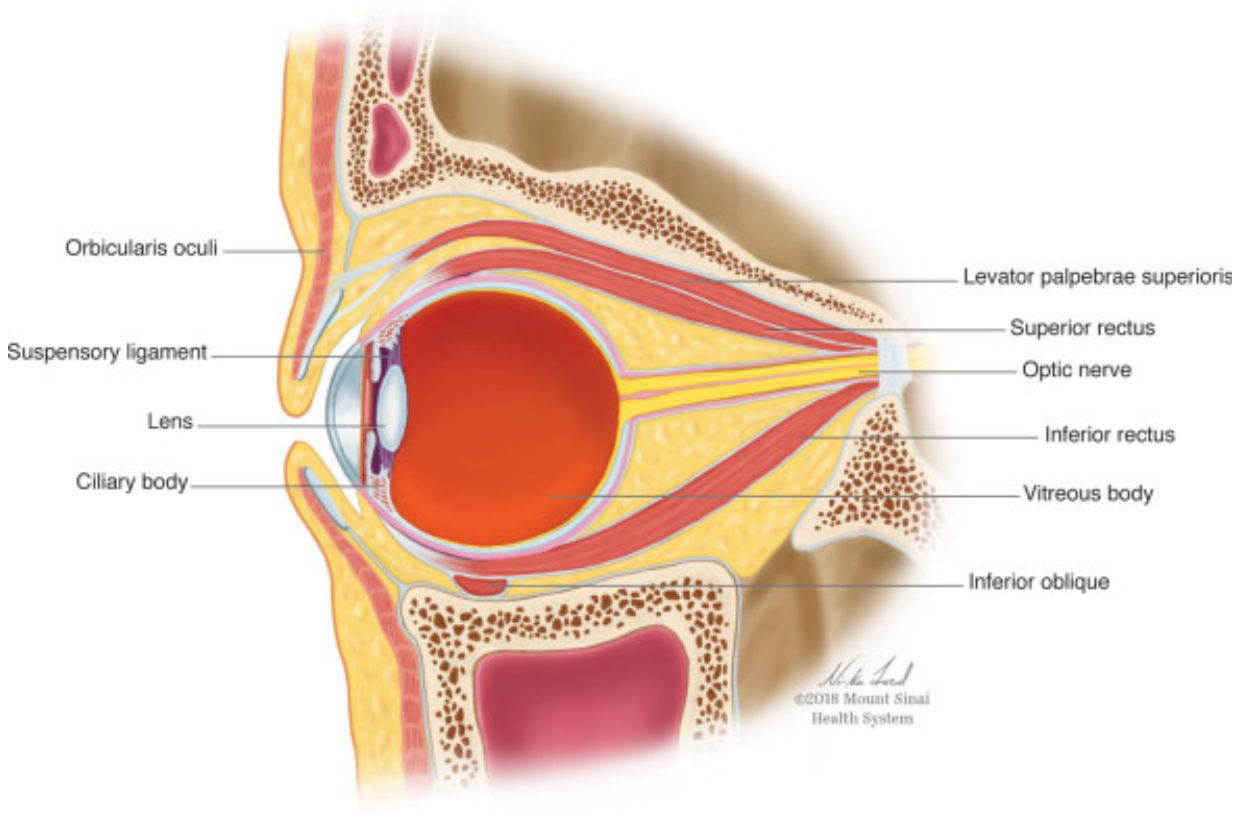

Fig. 4 Axial view of the extraocular ligaments of the orbit.

\section{Whitnall's Ligament}

Whitnall's ligament is a fascial sling that extends from the trochlea to the lateral orbital wall. It has attachments to the levator aponeurosis, superior rectus, conjunctiva, and Tenon's capsule.

\section{Check Ligaments}

The medial and lateral check ligaments extend from the orbital septum, levator aponeurosis, and muscle sheaths to attach to the medial orbital wall at the posterior lacrimal crest and lateral orbital wall at Whitnall's tubercle. ${ }^{14}$

\section{Orbital Fat}

Orbital fat is separated into the intraconal fat surrounding the optic nerve and the extraconal fat located between the rectus muscles and the periorbita. The fat is bordered anteriorly by the orbital septum and is organized into distinct fat pockets behind the septum. Posteriorly, the upper and lower lid fat pads are bordered by the levator aponeurosis or the capsulopalpebral fascia, respectively. In the upper eyelid, there are two fat pockets: nasal and central. In the lower eyelid, there are three compartments: nasal, central and temporal. These fat compartments are separated by the different septal and orbital fascias.

The intraconal portion of orbital fat contributes significantly to the maintenance of globe position. The extraconal fat is distributed throughout the anterior orbit and does not seem to contribute to the position of the globe. It is this latter fat that is reduced during blepharoplasty.

\section{Lacrimal System}

The lacrimal system is composed of the puncta, canaliculi, common canaliculus, lacrimal sac, and nasolacrimal duct.
The upper and lower puncta are located at the lid margin and travel to the canaliculi. The canaliculi extend superiorly or inferiorly from the puncta and then turn medially to parallel the eyelid margin. In most patients, the upper and lower canaliculi combine into a common canaliculus prior to entering the lacrimal sac. The valve of Rosenmüller is located at this point and prevents backflow of fluid from the lacrimal sac into the common canaliculus. The lacrimal sac is contained within the lacrimal sac fossa, which is comprised of the maxilla anteriorly and the lacrimal bone posteriorly. The fossa is found adjacent to the middle meatus at or just anterior to the head of the middle turbinate. The lacrimal sac then drains into the nasolacrimal duct, which finally empties into the inferior meatus at the valve of Hasner.

\section{Extraocular Muscles}

The extraocular muscles, which are responsible for eye movement, include the four rectus muscles, the superior oblique muscle, and the inferior oblique muscle. With the exception of the inferior oblique, all of the extraocular muscles originate at the annulus of Zinn and travel anteriorly to insert into the globe (-Fig. 5).

The levator superioris also originates at the annulus of Zinn, acts to elevate the upper eyelid, and is innervated by the oculomotor nerve (III). The superior oblique attaches via a trochlea to the orbit on the medial side of the roof, and its tendon extends posteriorly from the trochlea and laterally to insert onto the posterior globe. It functions to rotate the globe inferiorly and is innervated by the trochlear nerve (IV).

The inferior oblique attaches to the medial orbital rim and runs obliquely along the orbital floor over the inferior orbital fissure to attach to the globe. It rotates the eye externally and superiorly and is innervated by the oculomotor nerve (III). 


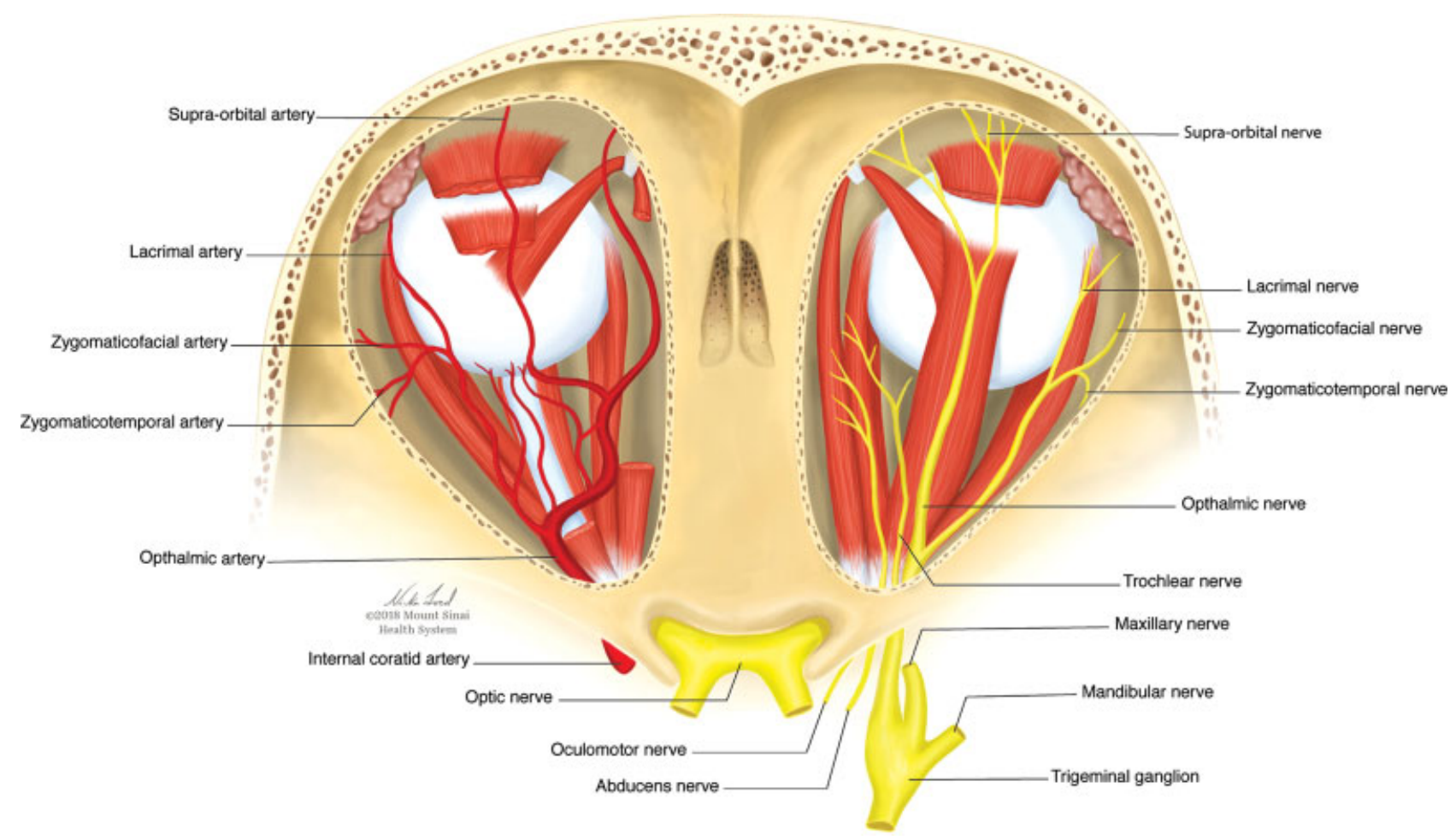

Fig. 5 Extraocular muscles and neurovascular supply of the orbit.

The superior, medial, inferior, and lateral rectus muscles run from the annulus of Zinn anteriorly to insert into the globe. The medial, inferior, and superior rectus muscles are innervated by the oculomotor nerve (III). The smooth muscle portion of the inferior and superior lid (Mueller's muscles) is supplied by sympathetic fibers of the superior cervical ganglion. The lateral rectus is innervated by the abducens nerve (VI). All of these nerves enter the orbit through the superior orbital fissure.

\section{Bulbus Oculi}

The ocular globe is an approximately spherical, hollow structure composed of a fluid-filled cavity surrounded by a wall made of three layers, an outermost fibrous layer, an intermediate vascular layer, and an innermost neural layer. The fibrous layer encompasses the sclera and cornea; the vascular layer consists of the uvea, which is composed of the iris, ciliary body, and choroid; and the neural layer is made up of the retina, which extends over the optic nerve and deep to the choroid around the walls of the globe to the ora serrata. This trilaminar structure further subdivides the globe into two compartments, the anterior compartment, which contains aqueous humor, and the posterior compartment, which contains vitreous humor.

The anterior compartment of the globe is bounded by the cornea anteriorly and the lens posteriorly. It is further divided into two chambers that communicate through the pupil, the anterior chamber, which is located between the cornea and the iris, and the posterior chamber, which lies between the iris and the lens. The posterior compartment of the globe is bounded anteriorly and posteriorly by the hyaloid membranes. The hyaloid canal projects from the posterior aspect of the lens to the center of the optic disc.

The vascular supply of the globe is provided by branches of the ophthalmic artery, specifically the central retinal artery and the short and long posterior ciliary arteries. Sensory innervation is from the long and short ciliary nerves, branches of the nasociliary nerve.

\section{Surgical Approaches to the Orbit}

Traditional approaches to the anterior orbit involve either transcutaneous or transconjunctival incisions. The location of the incision is determined by the quadrant of the orbit that needs to be accessed. Transcutaneous incisions, with the exception of the bicoronal approach, are made at the orbital rim or within a lid crease. Examples of orbital rim incisions include the direct brow, subbrow, inferior rim, Kronlein, and lateral canthotomy. Eyelid incisions include the upper lid crease, subciliary, and subtarsal, discussed briefly below. ${ }^{15}$

Exposure of the superior orbit can be achieved using a transcutaneous incision in the supratarsal fold of the upper lid. This incision is extended through the skin and orbicularis muscle. Subsequent dissection in the plane between the orbicularis oculi and orbital septum will allow entry into the orbit. Whether the periosteum or the orbital septum is incised depends on the surgical indication. For more extensive exposure of the superior orbit and/or the medial and lateral walls or to allow access for orbital osteotomies, the bicoronal incision is the preferred approach. An incision is made along the vertex of the head and extended inferiorly into either the preauricular or postauricular regions. Inferior dissection from the hairline to the orbital rims is then carried 
out in the subpericranial plane to avoid injury to the facial and supraorbital nerves.

For access to the inferior and lateral orbit, the most frequently utilized cutaneous approaches are the subciliary and subtarsal approaches. The subciliary incision is made approximately $2 \mathrm{~mm}$ below and parallel to the lash line. The preseptal plane can then be entered directly through the orbicularis or in a stepped manner and followed to the level of infraorbital rim. The periosteum along the anterior surface of the orbital rim is incised to provide access to the bony orbit. The subtarsal approach involves a transcutaneous incision in the subtarsal fold or 5 to $7 \mathrm{~mm}$ below the free lid margin. The orbicularis oculi is divided a few millimeters below the skin incision, and dissection is performed anterior to the orbital septum down to the infraorbital rim. Periosteum is again incised. ${ }^{16}$

The transconjunctival approach is useful for access to the inferior, lateral, and medial orbit. To achieve access comparable to the transcutaneous approaches, this approach may often need to be combined with a lateral canthotomy. The inferior fornix transconjunctival incision is made through the lower lid conjunctiva approximately 2 to $3 \mathrm{~mm}$ inferior to the tarsus. Dissection proceeds in either a preseptal or postseptal plane to the infraorbital rim, where the periosteum is incised to gain access to the orbital floor. The lower lid retractors and conjunctival incision do not need to be reattached. However, if a combined lateral canthotomy was performed, resuspension of the lower lid tarsal plate via the lateral canthal attachment is necessary.

Access to the posterior orbit or retrobulbar space may involve a lateral orbitotomy, which involves a lateral canthotomy or extended eyelid crease skin incision followed by removal of a portion of the lateral orbital wall.

To expand surgical access to the medial canthal apparatus, lacrimal drainage system, and/or inferior oblique muscle, a transcaruncular approach can be used either alone or in conjunction with the inferior fornix approach. The incision is located between the plica semilunaris and caruncle and extends into the superior and inferior fornices. Dissection is carried toward the posterior lacrimal crest, where the periorbita is incised to allow access to the medial orbit. The medial orbit may also be approached transcutaneously through a curvilinear incision anterior to the medial canthus that extends along the frontal process of the maxilla superiorly to the nasofrontal suture and inferiorly to the orbital rim. Adequate exposure can be limited by the medial canthal ligament to the frontal process. If the medial canthus is detached, it must be carefully resuspended, usually via a transnasal canthoplexy. ${ }^{17}$

Neurosurgical approaches, such as the fronto-orbitozygomatic cranio-orbitotomy, can provide a global view of the deep and medial components of the orbit, including the orbital apex, optic canal, and adjacent intracranial structures. ${ }^{18}$

More recently, endoscopic approaches to the orbit have been developed and described in the surgical treatment of both extraconal, particularly for the orbital apex and periorbital skull base, and intraconal lesions. Several studies have shown similar efficacy and complication rates compared to traditional external approaches. ${ }^{19}$ Specifically, transorbital neuroendoscopic surgery has also been developed to allow for access to difficult-to-reach areas such as the greater wing of the sphenoid and the lateral portion of the anterior skull base.

\section{Conflicts of Interest}

None declared.

\section{References}

1 Ochs MW, Buckley MJ. Anatomy of the orbit. Oral Maxillofac Surg Clin North Am 1993;5:419-429

2 Tessier P, Rougier J, Herrouat F, et al. Surgical anatomy of the orbit. In: Wolfe SA, translator. Plastic Surgery of the Orbit and Eyelids: Report of the French Society of Ophthalmology. New York, NY: Masson Publishing; 1981

3 Hollingshead WH. Anatomy for Surgeons. Vol. 1. Philadelphia, PA: Harper and Row Publishers; 1982

4 Romanes GJ. Cunningham's Textbook of Anatomy. Vol. 10. London, UK: Oxford University Press; 1964

5 Rontal E, Rontal M, Guilford FT. Surgical anatomy of the orbit. Ann Otol Rhinol Laryngol 1979;88(3 Pt 1):382-386

6 Chastain JB, Sindwani R. Anatomy of the orbit, lacrimal apparatus, and lateral nasal wall. Otolaryngol Clin North Am 2006;39(05): 855-864, v-vi

7 Lang J. The floor of the orbit. In: Lang J, ed. Clinical Anatomy of the Head. Berlin, Germany: Springer Publishing; 1981

8 Jeong SM, Park KJ, Kang SH, et al. Anatomical consideration of the anterior and lateral cutaneous nerves in the scalp. J Korean Med Sci 2010;25(04):517-522

9 Beer GM, Putz R, Mager K, Schumacher M, Keil W. Variations of the frontal exit of the supraorbital nerve: an anatomic study. Plast Reconstr Surg 1998;102(02):334-341

10 Abed SF, Shams P, Shen S, Adds PJ, Uddin JM, Manisali M. A cadaveric study of the cranio-orbital foramen and its significance in orbital surgery. Plast Reconstr Surg 2012;129(02):307e-311e

11 Robinson TJ, Stranc MF. The anatomy of the medial canthal ligament. Br J Plast Surg 1970;23(01):1-7

12 Jordan DR, Mawn L, Anderson RL. Surgical Anatomy of the Ocular Adnexa: A Clinical Approach. 2nd ed. New York, NY: Oxford University Press; 2012:232

13 Ansari MW, Nadeem A. Anatomy of the eyelids. In: Ansari MW, ed. Atlas of Ocular Anatomy. Cham, Switzerland: Springer Publishing; 2016

14 Lockwood CB. The anatomy of the muscles, ligaments, and fasciae of the orbit, including an account of the capsule of Tenon, the check ligaments of the recti, and the suspensory ligaments of the eye. J Anat Physiol 1885;20(Pt 1):i2-i25

15 Khan AM, Varvares MA. Traditional approaches to the orbit. Otolaryngol Clin North Am 2006;39(05):895-909, vi

16 Rohrich RJ, Janis JE, Adams WP Jr. Subciliary versus subtarsal approaches to orbitozygomatic fractures. Plast Reconstr Surg 2003;111(05):1708-1714

17 van der Meulen JC, Gruss JS. Ocular Plastic Surgery. London: Mosby-Wolfe; 1996

18 Maroon JC, Kennerdell JS. Surgical approaches to the orbit. Indications and techniques. J Neurosurg 1984;60(06): 1226-1235

19 Campbell AA, Grob SR, Yoon MK. Novel surgical approaches to the orbit. Middle East Afr J Ophthalmol 2015;22(04):435-441 